\title{
ADAMANTIUM Project: Enhancing IMS with a PQoS-aware Multimedia Content Management System
}

\author{
Harilaos Koumaras ${ }^{1,3}$, Daniel Negrou ${ }^{2}$, Fidel Liberal ${ }^{3}$, Javier Arauz ${ }^{4}$, Anastasios Kourtis ${ }^{1}$ \\ ${ }^{1}$ NCSR Demokritos, Institute of Informatics and Telecommunications, Greece \\ \{koumaras, kourtis\}@iit.demokritos.gr \\ ${ }^{2}$ Viotech Communications, France \\ daniel.negru@viotech.net \\ ${ }^{3}$ University of the Basque Country, Department of Electronics and Telecommunications, Spain \\ fidel.liberal@ehu.es \\ ${ }^{4}$ Ericsson, Spain \\ jesus.javier.arauz@ericsson.com
}

\begin{abstract}
IMS entails novel business opportunities for pioneering and emerging multimedia services, such as IPTV and VoIP video call applications. However, this strong commercial interest on this promising convergent IMS environment is balanced by the lack of efficient user/customer-centric network management mechanisms. ADAMANTIUM proposes an IMS-compatible Multimedia Content Management System (MCMS) focused on performing dynamic cross layer adaptations for optimization of the user experience in terms of perceptual quality for media services. This multimodal management system will be applied in an integrated and coherent way along all the network layers and delivery-chain nodes based on a user/customer-centric approach rather than a typical engineering one.
\end{abstract}

\section{INTRODUCTION}

Fixed/mobile convergence is a massive trend that requires adequate network and service infrastructures. One of the visions of this trend is that services will be sold in a consumer mass market based on the provision of content at a requested quality, exploiting the Perceived Quality of Service (PQoS) concept [1]. The evaluation of the PQoS for media content that have variable bandwidth demands, will provide a user with a range of potential choices covering for example the possibilities of low, medium or high quality levels, indication of service availability and costs. However, the IMS infrastructure [2-3] the most promising platform for the convergence- currently does not consider any Perceived QoS-aware management mechanism within its service provision management system, eliminating its traffic policies to the UMTS SBLP, which is a typical traffic differentiation mechanism that classifies the service bearers to different classes with specific QoS constraints.

In this brewing environment, ADAMANTIUM project, going beyond the current state-of-the-art and paving the way to the future interaction between Multimedia Services and IMS, proposes an extension of the IMS management capabilities through PQoS-aware mechanisms. More specifically, it is introduced the concept of PQoS awareness into the current IMS management system, towards which the whole traffic engineering is not performed abruptly, but with scope the PQoS optimization at the user terminal.
More specifically ADAMANTIUM proposes an IMS compatible PQoS-aware multimedia content management system, which in case of possible distorted playback of the multimedia service at the users terminal, will be able to dynamically and in real time adapt the various NQoS parameters across the layers (i.e. service, network and link) of the network delivery chain (i.e. service generation, delivery and consumption) with scope the optimization/maximization of the delivered PQoS level and as such not interfering with the end-user experience of the content being consumed [4]. Moreover, the proposed management system does not only improves abruptly the delivered PQoS level but it performs a sophisticated reallocation of the occupied resources in order to keep the total traffic of the bearer constant, indicating that the proposed management system uses more efficiently the already utilized resources, without requesting/spending more resources in order to perform the PQoS optimization.

Hence, the marketing perspectives of the ADMAANTIUM MCMS are multidimensional creating new and sustainable market opportunities based on converged business models between the telecom, the content and the consumer industries.

From the operator's aspect, ADAMANTIUM manages more efficiently the allocated resources to the adapted services without altering the total service/bearer bit rate, providing therefore an optimal trade-off between service quality provision and resource consumption.

From the customer's perspective, which pays for gaining access to a specific media service, ADAMANTIUM enhances and maximizes the user experience in terms of perceptual constraints without any extra cost or charge. Therefore, ADAMANTIUM adds value to the promotion of the emerging multimedia services over an error prone environment, reassuring a cost-effective and perceptually acceptable service reception.

Following this introductory section, the rest of the paper is organized as follows: Section II describes the ADAMANTIUM overall architecture, Section III presents the proposed Multimedia Content Management System (MCMS) and Section IV 
describes a typical case scenario. Finally Section V concludes the paper.

\section{ADAMANTIUM OVERALL ARCHITECTURE}

Figure 1 depicts the overall architecture of ADAMANTIUM project. It comprises of:

- The ADAMANTIUM Multimedia Content Management System (MCMS) Module, which is the main project entity and focuses on monitoring the network statistics (i.e. core, access, terminal), the service generation (i.e. Media Server Resource Function (MSRF), VoIP terminals) and the service perceptual level at the end-user terminal in order to define and apply an optimal cross layer adaptation action across the delivery network chain and media lifecycle (i.e. service generation node, core network, access network and end-user terminal) for maximizing the user satisfaction.

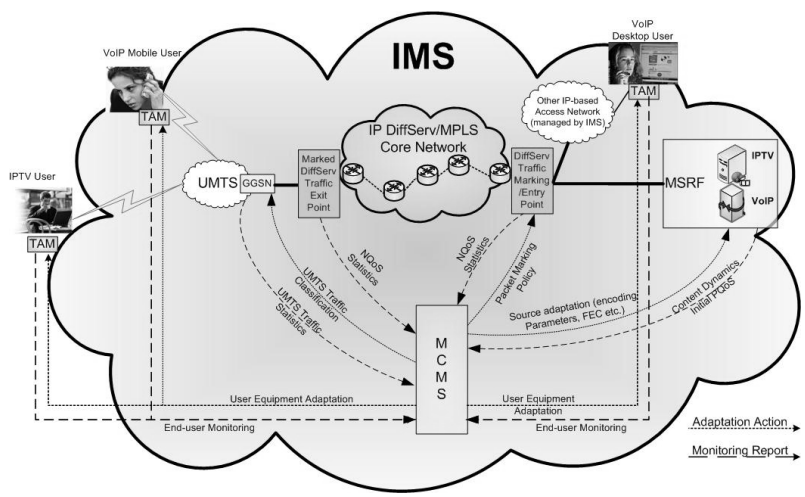

Figure 1. The overall architecture of ADAMANTIUM

- The ADAMANTIUM Multimedia Server and Resource Function (MSRF), which is an IMS-based MRF module with an additional threefold responsibility:

- The IPTV service generation, session management and service streaming.

- The VoIP service session management and signaling.

- The monitoring of the spatial and/or temporal content dynamics along with the selected encoding/streaming parameters

- The adaptation, according to the MCMS commands, of the IPTV encoding parameters and/or the respective FEC value.

\section{- IP Core - DiffServ/MPLS Core Network}

ADAMANTIUM considers a DiffServ/MPLS-enabled core network for the delivery of the requested multimedia service. The MCMS will be equipped with compatible modules and interfaces for the packet marking and traffic monitoring at the edges of the DiffServ/MPLS traffic network, considering all the core network nodes (i.e. routers) as inter-cooperated and capable of treating appropriately marked traffic by the corresponding DiffServ/MPLS mechanisms, without being necessary any further configuration by the IMS/MCMS.

\section{- The UMTS Access Network}

At the access side a UMTS access network is considered, which provides service/bearer classification mechanisms for providing QoS constraints on the delivered service type (e.g. video, voice, data, etc). ADAMANTIUM project will exploit these classification mechanisms of the GGSN in its sophisticated PQoS-aware cross layer adaptation procedure with scope the perceptual optimization of the delivered service. Moreover, in order to support interactive multimedia services, the Multimedia Broadcast/Multicast Service (MBMS) infrastructure will be considered as an extension to the typical UMTS architecture, which provides an uplink channel for the provision of interactive and bidirectional applications.

- The ADAMANTIUM Terminal Adaptation Module (TAM). At the PQoS-enabled user terminals (e.g. 3G mobile handset, SIP voice/video phone (hardphone or softphone), PDA) a TAM will be integrated, which enables the terminal's interaction with the appropriate interfaces/modules of the MCMS. ADAMANTIUM concept aims to be applicable to a mass market of mobile terminal devices and therefore the TAM technical requirements will be minimal in order to be straightforward applicable to future commercial mobile handsets.

There are two functions associated between the MCMS and the TAM of the PQoS-enabled user terminals:

- Adaptation function: according to the control command/parameters received from the MCMS, the PQoSenabled user terminal adapts on the fly its VoIP/Videocall codec, encoding bit rate or mode, packetization scheme (e.g. numbers of frames in a packet) and jitter buffer size (or buffer algorithm if required), aiming at end-to-end perceived quality improvement.

- Monitoring function: reporting delivered PQoS (e.g. MOS score for voice and video) and relevant terminal parameters (e.g. codec type, bit rate, encoding mode, packetization parameters) to the MCMS module. These parameters are obtained by the TAM interface, which is implemented in the terminal device.

When the monitoring PQoS value at the mobile terminal remains for a specific temporal duration below a pre-defined perceptual threshold, a PQoS alarm will be triggered back to the MCMS, which will in turn initiate the sophisticated ADAMANTIUM cross layer adaptation procedure. This predefined perceptual threshold corresponds to the lowest acceptable perceived quality level and is dependent on the application/service type and the user profile

Towards minimizing false initiations of the adaptation procedure, combined PQoS and CNR statistics will be considered into triggering this PQoS feedback mechanism. Perceptual sub- 
jective studies will be exploited for defining the optimal theoretical and statistical trade-off between the monitored perceptual degradation, its temporal duration and the respective CNR (Carrier-to-Noise Ratio) value.

Finally, it is essential to stress on the inter-dependent dynamic cross layer nature of the proposed adaptation by the MCMS module, according to which the adaptation actions are performed without altering the total traffic of the service bearer. Towards this, considering an adaptation at the media server (e.g FEC change) in order to enhance the service robustness and subsequently the delivered PQoS level, then a respective adaptation of the encoding/streaming parameters must be also considered in order to retain constant the total service bit rate. Thus, the MCMS will dynamically and inter-dependently adapt all the relative QoS-related parameters at every step of the media lifecycle (i.e. from the service generation to content consuption), following a cross layer method (i.e. from the service to link layer), in order to maximize the delivered PQoS level with simultaneous maintenance of the total service/flow/bearer traffic.

\section{THE MULTIMEDIA CONTENT MANAGEMENT SYSTEM}

The ADAMANTIUM project is based on a Next Generation Network IMS infrastructure and aims at developing within this NGN environment an integrated PQoS-aware management system, which will be able to maximize in real time the end user satisfaction for the cases that PQoS degradation is noticed. This system is called Multimedia Content Management System (MCMS) and will perform dynamic and content dependent cross layer adaptation techniques across the multimedia network delivery chain.

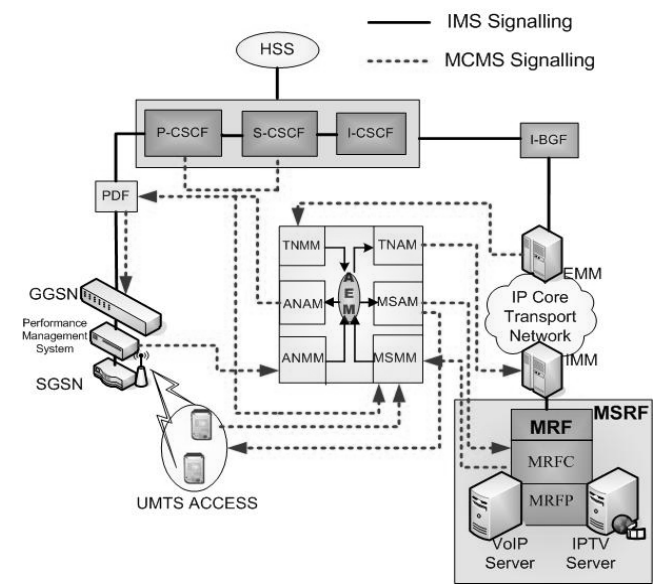

Figure 2. The ADAMANTIUM MCMS and NGN IMS architecture

The MCMS is the central entity of the proposed PQoS-aware and user-centric ADAMANTIUM IMS architecture that will seamlessly communicate with the already existing IMS modules and interfaces. Thus, MCMS will be fully IMS compliant, composed of modules based on IMS signaling (preferably SIP/SDP, although Diameter will be also considered) for communication, processing and interaction with the rest IMS modules and interfaces, although IMS Diameter will also be possi- ble. Figure 2 depicts the overall architecture of the proposed MCMS within the IMS-native components, their signaling relations and interactions.

According to figure 2, the IMS Application Servers (AS) for IPTV and VoIP applications are hosted within the Media Resource Function (MRF), which executes IMS applications and services by manipulating SIP and SDP signaling for interfacing with other systems. ADAMANTIUM project considers that MRF and AS modules are combined into a single entity, which will be called as Media Server Resource Function (MSRF). The MSRF, besides being a media server, provides mechanisms for bearer-related services such as conferencing or bearer transcoding, through a controller (MRFC) and a processor (MRFP) in compliance with the MCMS decisions.

The HSS (Home Subscriber Server) is the master user database that supports the IMS network entities that are actually exploited by the CSCF modules for handling the calls/sessions. The HSS contains the subscription-related information (user profiles), performs authentication and authorization of the user, and can provide information about the physical location of user.

The control layer of the IMS infrastructure consists of nodes for managing call establishment, management and release, which are called Call Session Control Functions (CSCF). The CSCF inspects each SIP/SDP message and determines if the signaling should visit one or more application servers en route to its final destination. More specifically, the CSCF is a distributed entity comprised of three different components:

- The Proxy CSCF (P-CSCF), which acts as the entry point for any service invocation within IMS and grants appropriate access rights after successful user authentication. The P-CSCF is tasked to relay session and media related info through Diameter protocol to the Policy Decision Function (PDF) when the operator wants to apply Service Based Local Policy (SBLP). Based on the received information the PDF is able to derive authorized NQoS information that will be passed to the GGSN when SBLP is applied.

- The Interrogating CSCF (I-CSCF) acts as a topologyhiding gateway between the P-CSCF and the S-CSCF, by determining the S-CSCF or the AS to which an end-user should register. I-CSCF is a contact point within an operator's network for all connections destined to a subscriber of that network operator. The I-BGF (Interconnection Border Gateway Function) will be also used as gateway to IP networks, providing NAT and Firewall functions;

- The Serving CSCF (S-CSCF) is responsible for key routing decisions as it receives all the User Equipment (UE)-originated and UE-terminated sessions and transactions. Therefore it is also responsible for handling registration processes, maintaining session states and storing the service profiles.

In this multimodal management environment the MCMS modules comes to enhance the current IMS management capabilities by adding real-time dynamic cross layer adaptation pro- 
cedures for providing end-to-end perceptual optimization and therefore maximization of the user experience.

The MCMS architecture will be based on a central decision module, the Action Engine Module (AEM), responsible for taking optimal adaptation decisions based on the monitoring of network and perceptual statistics, gathered by IMS-based (preferably SIP/SDP-based) monitoring and adaptation modules. Afterwards, the AEM exploiting theoretical mapping frameworks between NQoS and PQoS will process all the selected statistics and define a perceptually optimal cross layer adaptation action.

More specifically, for monitoring purposes the following modules are considered:

\section{- Multimedia Service Monitoring Module (MSMM)}

The MSMM performs monitoring of the service session through the P-CSCF and S-CSCF modules of the IMS. This is the only module of the MCMS that is activated when a service is requested; whilst all the rest remain in idle mode until the active adaptation procedure starts. Then, the MSMM, except from simply informing the MCMS for the liability of the service, monitors the PQoS and CNR values at the end-user mobile terminal device, while at the service generation site, the content dynamics and the encoding parameters are also monitored through the TAM of the terminal device.

\section{- $\quad$ Transport Network Monitoring Module (TNMM)}

The TNMM module is used during the dynamic cross layer adaptation procedure for monitoring network statistics like packet loss, jitter etc. at the DiffServ/MPLS core transport network. Towards this, the appropriate External Marking Modules (EMM) interface will be developed and integrated at the egress edge router of the core transport network for enabling interaction and communication with the TNMM of the MCMS.

\section{- Access Network Monitoring Module (ANMM)}

The ANMM module monitors the UMTS access network statistics, based on the exploitation of historical data, which are updated in near real time by the UMTS performance management system at frequent time periods.

For adaptation purposes, MCMS considers the following modules, through which the optimal adaptation actions for PQoS optimization are applied:

\section{- Multimedia Service Adaptation Module (MSAM)}

The MSAM performs adaptation actions at the end-user terminal device and at the service generation entity (i.e. the MSRF for IPTV services or the end-user terminal for VoIP applications) relative to the decoding (i.e. buffer scheme) and encoding (bit rate, packetization etc.) parameters, as well as FEC value adaptation towards enhancing the error resilience of the delivered service

\section{- $\quad$ Transport Network Adaptation Module (TNAM)}

The TNAM applies the adaptation actions to the DiffServ/MPLS-enabled core transport network through the Internal Marking Module (IMM), which will be developed and in- tegrated at the ingress router of the core network. The IMM receives the adaptation actions from the TNAM and translate them to DiffServ/MPLS compatible commands, which are finally applied by marking appropriately the incoming traffic.

\section{- $\quad$ Access Network Adaptation Module (ANAM)}

The ANAM applies the adaptation actions, decided by the AEM of the MCMS, to the UMTS access network through the IMS PDF module. The PDF in turn applies them at the GGSN by performing service bearer classification in order to improve its QoS characteristics and therefore enhance the delivered PQoS level.

\section{A. MCMS Interaction with Access Network Modules}

At the access network, UMTS offers tele-services and bearer services by supporting both connection-oriented and connectionless services for Point-to-Point and Point-to-Multipoint communication. The proposed architecture will focus on the packet switched elements of UMTS, such as the Serving GPRS Support Node (SGSN) and the Gateway GPRS Support Node (GGSN).

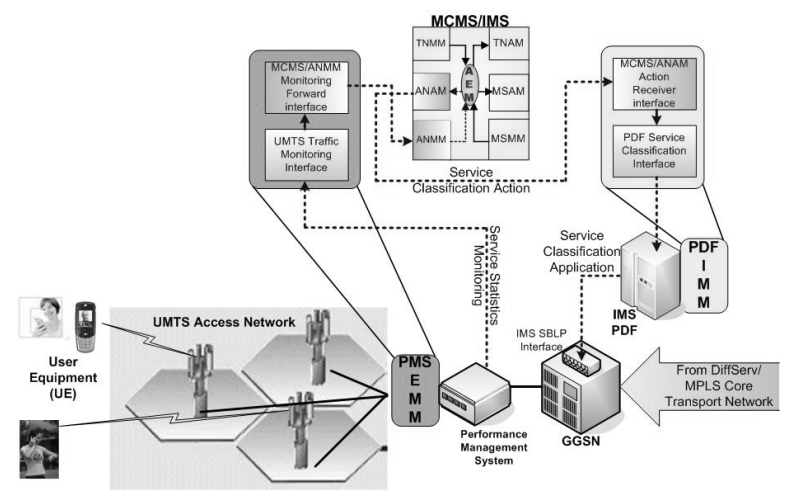

Figure 3. The ADAMANTIUM Access Network Architecture

Figure 3 depicts the proposed Access Network architecture, where monitoring and adaptation activities will be focused on extending the existing IMS Service Based Local Policy (SBLP) mechanisms at the access domain by specifying and developing the appropriate interfaces for communication between i) the ANAM/MCMS and the IMS/PDF ii) the ANMM/MCMS and the UMTS Performance Management System (PMS).

More specifically, for monitoring purposes it will be specified the UMTS External Monitoring Module (EMM), which will interact using IMS-based messages (e.g. SIP/SDP) with the UMTS PMS for providing near real time access network statistics to the ANMM/MCMS. Similarly, for adaptation purposes the PDF Internal Marking Module (IMM) will be developed for communication between the ANAM/MCMS and the IMS/PDF entity, through which the traffic/service classification will be applied to the GGSN, based on the existing IMS SBLP differentiation mechanisms.

\section{B. MCMS Interaction with Core Network Modules}

Similarly, it will be designed, developed and demonstrated a core network architecture, which mixes the capabilities of Differential Services and Multiprotocol Label Switching technolo- 
gies with final scope the best efficiency of a network which carries IPTV and VoIP services. Towards this, the typical DiffServ/MPLS network architecture will be enhanced by integrating them with the MCMS/IMS adaptation procedures, which will enhance the policy decision mechanisms by considering PQoS-aware traffic classification.

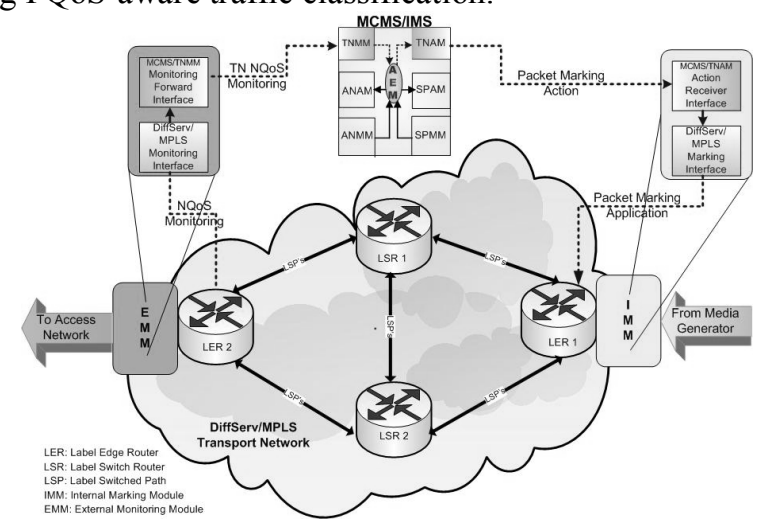

Figure 4. ADAMANTIUM DiffServ/MPLS Core Network Architecture

Figure 4 depicts the proposed DiffServ/MPLS transport network architecture where the DiffServ/MPLS Internal Marking Module (IMM) and DiffServ/MPLS External Monitoring Module (EMM) modules will be developed for the interaction between the MCMS relative modules (i.e. TNMM and TNAM) and the DiffServ/MPLS ingress and egress routers.

Once the AEM of the MCMS has determined a specific adaptation action for improving/optimizing the delivered PQoS level, then regarding the DiffServ/MPLS transport network, the appropriate IMS-based (e.g. SIP/SDP-based) adaptation action is forwarded from the TNAM/MCMS module to the MCMS/TNAM Action Receiver interface of the Internal Marking Module (IMM), which initially translates the received adaptation action into standard DiffServ/MPLS classification commands. These translated actions are further forwarded to DiffServ/MPLS Marking interface of the IMM, which applies them into the ingress router (i.e. LER 1) of the transport network, where the actual packet marking and traffic classification is performed through the relative labels of the incoming traffic.

Then the marked/classified media flow is treated appropriately by the mechanisms of the DiffServ/MPLS-enabled Label Switch Routers (LSRs) across the transport network, minimizing -according to the characteristics of each traffic class- the effects of the network delay, packet loss etc. on the streaming flow of the IPTV/VoIP service.

At the other end, where the media flow exits the coretransport network through the egress router (i.e. LER 2), the External Monitoring Module (EMM) will calculate network related statistics about the jitter, packet loss etc of each classified flow. These NQoS-related statistics will be calculated by the DiffServ/MPLS Monitoring Interface of the EMM and afterwards they will be forwarded to MCMS/TNMM Monitoring Forward Interface, which translates them into MCMScompliant form and finally reports them into the TNMM module of the MCMS. According to the reported statistics from the TNMM, and taking under consideration the monitoring statis- tics from the MSMM and ANMM, the AEM/MCMS will decide the optimal adaptation action for improving the PQoS level of the delivered IPTV applications.

\section{MCMS Interaction with MSRF and Terminals}

The adaptation of the multimedia content according to the changing usage environments during the service delivery is becoming more and more important, because the characteristics of the environment (e.g., Quality of Reception) are varying during the consumption of the multimedia service. Thus, immediate actions are needed to be performed in order to enable a unique, worthwhile, and seamless multimedia experience during the entire session. These actions are usually referred as dynamic multimedia content adaptation.

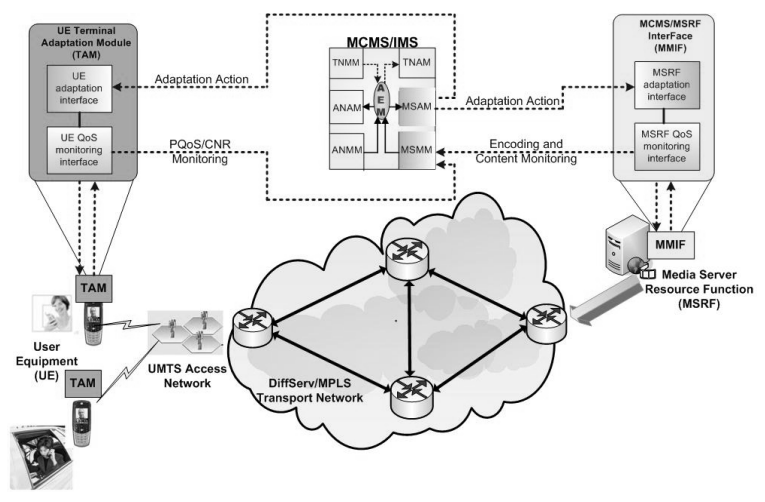

Figure 5. The Modules Interaction for IPTV Service Adaptation

To this end, for realizing these service adaptation tasks, the MCMS needs to interact with the two end-point entities: the MSRF and the Terminal Adaptation Module (TAM) of the User Equipment, for adaptation actions and PQoS monitoring. Figure 5 depicts the overall interaction mechanism for the case of IPTV service adaptation. When the MCMS is running in active mode, specific sub-modules/interfaces of each entity are in charge of performing these tasks:

- At the MCMS side, the two sub-modules having a specific role for service adaptation are: i. The MSMM (Multimedia Service Monitoring Module), which receives the MS PQoS, $\mathrm{CNR}$, content dynamics and the encoding statistics monitoring information from both the MSRF and the TAM of the end user. ii. The MSAM (Multimedia Service Adaptation Module), which forwards the MS adaptation actions to be undertaken by the MSRF and/or TAM according to the AEM PQoS-aware adaptation decision.

- At the UE and at the Media Server sides, two different interfaces are introduced for interacting with the MCMS and acting accordingly for the service adaptation and monitoring: $i$. The Terminal Adaptation Module at the User Equipment (UE/TAM) ii. The MSRF/MCMS InterFace (MMIF) located at the MSRF

The UE/TAM interface is a hybridic interface installed on the end-user mobile handset, performing either monitoring or adapting actions, where the monitoring includes the PQoS for the delivered video quality of IPTV and the CNR value, indica- 
tive of the quality signal reception at the physical layer and the adaptation is relative to the coding adaptation for VoIP services

\section{CASE SCENARIO}

Considering a requested multimedia service at a specific PQoS level by a mobile handset with TAM integrated, the first contact point within the IMS infrastructure is the P-CSCF module, which relays the services session. Then the P-CSCF interacts with the MSMM of the MCMS messages based on IMS protocols (eg. SDP/SIP or Diameter), reporting the request of the specific service. Since a session has been established for the specific service, the MSMM/MCMS communicates with the S-CSCF, which maintains the service session and informs the MCMS about the session viability. This idle mode of the MCMS is essential for its scalability abilities, because during this operating mode, resources are not consumed. The MCMS simply monitors through the S-CSCF that the service session remains active, awaiting the reception of a PQoS-degradation alarm from the end-user terminal, indicating poor perceptual quality and therefore bad user experience.

During the service session, at the end-user mobile handset through a ultra light software application, the PQoS level and the CNR values are internally monitoring in real time, along with the duration that the measured PQoS is below a specific perceptual threshold. The choice of these two monitoring values is representative of the service and physical QoS status, providing complementary information about the cause of the monitoring PQoS degradation. In this framework, the intelligent PQoS-aware mobile handset when monitors a PQoS degradation in conjunction with bad signal reception (i.e. High CNR value), then the TAM of the mobile handset will not trigger a PQoS alarm to the MCMS, because this perceptual degradation is possibly caused by poor signal reception due to the location of the user. Thus, false alarm triggering is avoided, saving system resources and unnecessary use of the MCMS.

If PQoS degradation below a specific perceptual threshold and for given duration is observed at the PQoS-aware intelligent end-user terminal in conjunction with low $\mathrm{CNR}$ value (i.e. good reception, showing that the cause of the PQoS degradation is NQoS-related), then a PQoS alarm is triggered through the TAM interface of the terminal back to the MSMM, which till that moment passively monitors the session. Then, the idle mode of the MCMS for the specific session is terminated, which means that the dynamic cross layer adaptation procedure is initiated and all the monitoring and adapting modules of the MCMS are activated for the specific session. For all the rest on-going sessions, which do not face PQoS degradations, the MCMS continues to operate in idle mode, reassuring minimal resources consumption and enabling large-scale scalability abilities for MCMS.

Once the MCMS has been switched to active mode, the current QoS relative statistics across the network delivery chain (i.e. from the core and access network) are reported to MCMS via the ANMM and TSMM modules. Also, reports from the encoder about the coding parameters, the content dynamics of the multimedia service, the current FEC scheme (if applies) and the decoding prameters at the terminal (i.e. buffer scheme) are reported to the MCMS via the MSMM. If VoIP session is considered, then the MSMM monitoring takes place at both the end-user terminals that participate in the session, where the voice/video is encoded and decoded at the sender and receiver terminal respectively. All the collected statistics will be further exploited by the MCMS, through a sophisticated processing procedure and a decision algorithm. This sophisticated algorithm will be implemented in the MCMS Action Engine Module (AEM), which will be the main processing unit of MCMS and will decide the appropriate actions and adaptations across the network delivery chain, in order to optimize the delivered PQoS, but maintaining constant the total service traffic.

After the monitoring phase of the network statistics across the delivery chain has been completed, the adaptation phase is following. This is performed through the adaptation action modules of the MCMS (i.e. the MSAM, TNAM, ANAM) and may include adaptation of the encoding parameters, the packetization schemes or the FEC scheme in order to enhance the error resilience of the multimedia service, along with DiffServ/MPLS and UMTS traffic policies through more QoSsensitive traffic/service classes. Towards this, the AEM will process all the received statistics by the ANMM, TPMM and MSMM in order to define the adaptation actions across the network delivery chain, aiming at the optimization of the NQoS, which will lead to maximization of the user satisfaction and the delivered PQoS level, without altering the total service traffic of the bearer. The AEM shall be able to take QoS control decisions based on an advanced mechanism/algorithm (e.g. such as neuronal, genetic programming or expert system), taking into account multiple and interrelated PQoS models.

\section{CONCLUSIONS}

Being harmonized with market needs, ADAMANTIUM will create the conditions for a new generation of media technologies, providing significantly higher performances through PQoS-aware dynamic cross layer adaptation techniques in the IMS infrastructure. By enhancing the existing IMS purely engineering management techniques with intelligent and flexible cross layer adaptation methods, higher performance at the provision of a new generation of media services will be offered.

\section{ACKNOWLEDGMENT}

The work in this paper has been performed within the research framework of FP7 ICT-214751 ADAMANTIUM Project.

\section{REFERENCES}

[1] H. Koumaras, A. Kourtis, D. Martakos, J. Lauterjung, "Quantified PQoS Assessment Based on Fast Estimation of the Spatial and Temporal Activity Level," Journal of Multimedia Tools and Applications, 34(3), 355 374, March 05, 2007.

[2] M. Poikselka, G. Mayer, H. Khartabil, A. Niemi, "The IMS IP Multimedia Concepts and Services", Wiley, 2006.

[3] G. Camarillo, M. A. Garcia-Martin "The 3G IP Multimedia Subsystem”, Wiley, 2004.

[4] H. Koumaras, A. Kourtis, C-H Lin, C-K Shieh, "A Theoretical Framework for End-to-End Video Quality Prediction of MPEG-based Sequences", The Third Inter. Conf. on Networking and Services - ICNS07, Athens, Greece, June 19-25 2007. 